\title{
3D Orchard Canopy Architectural Modelling for Use in Airflow and Drift Predictions
}

A. Melese Endalew, M. Hertog, P. Verboven, M.A. Delele, K. Baetens, H. Ramon and B.M. Nicolaï

BIOSYST-MeBioS, Katholieke Universiteit Leuven

Willem de Croylaan 42, B-3001 Leuven

Belgium

Keywords: orchard canopy, canopy architecture, airflow

\begin{abstract}
The current trend in studying the interaction of vegetation canopies with their environment and numerical prediction of drift is mainly based on porous media approaches. The methods involve several approximations and estimations that give the global effect of the canopies to airflow and drift without investigating the detailed local effects of the vegetation elements. These approaches also require precise estimation of certain parameters such as drag coefficient and leaf area density. To make some advances in the field and to address some of the above problems a new approach needed to be developed, where real canopy architecture was modelled and linked to a computational Fluid Dynamics (CFD) software to model airflow through the canopies. This helped to investigate the real effects of the vegetation elements on atmospheric airflow which directly affects drift and drift prediction. In this work, 3D orchard canopy architecture was modelled using a combined discrete-continuous plant growth simulation model, which considered the phenomenological plant growing behaviour and the effect of temperature. Two canopy geometries were introduced into a fluid domain and the domain was meshed. Airflow around and through the canopy was simulated using the Reynolds-averaged Navier-Stokes (RANS) equations and $k-\varepsilon$ turbulence model. The airflow simulation results agreed both qualitatively with wind tunnel validation experiment and quantitatively with previous works done in the area, ensuring the prospect of the architectural modelling for further application. It was also possible to show the detailed effects of canopy elements on airflow.
\end{abstract}

\section{INTRODUCTION}

High costs for plant protection and intensified concerns of the environment brought the need to improve spraying efficiency to guarantee maximum protection with minimum drift. One of the factors that contribute to spraying inefficiencies in orchard fields is related to a complex interaction of airflow and tree architecture. The spatial and temporal distribution of air velocity and turbulence within orchard canopies has to be well known to accurately predict the transport and deposition of pesticides. But the strongly complex three-dimensional, inhomogeneous and turbulent nature of airflow within the canopy and the complex nature of the tree architecture limited the process of accurate prediction. Consequently, porous medium models were adopted in which the plant canopy is averaged into a lumped volume to remove flow details associated with individual plant elements (Xu et al., 1997). Nearly in all of these models horizontal homogeneity, neutral stratification and steady-state conditions were assumed where the effects of plants and their elements were approximated by leaf area density, drag coefficient and sheltering factor (Thom, 1971). However, schematisation of a homogeneous medium cannot address the heterogeneous nature of canopies (Birch et al., 2003).

In recent years, there are many approaches to describe the geometric structure of plants in 3D. Some of them are structural models (Godin, 2000), functional structural models (e.g. L-systems) (Prusinkiewicz and Lindenmayer, 1996; Sievänen et al., 2000) and visualization and digitization methods (Sinoquet et al., 1997; Costes et al., 1999). Reviews of some plant architectural modelling are given by De Visser et al. (2002) and 
Prusinkiewicz (1998). Most of the approaches are intended for a specific application and some of them are even for a particular type and species of plants. Furthermore, little or no efforts were made to link these architectural models with CFD to simulate air and particle or pollutant transport within the canopies. This is the point where $3 \mathrm{D}$ orchard canopy architectural modelling was required. The objective of this work was, therefore, to overcome some of these limitations by developing 3D orchard canopy architectural modelling that could be linked with CFD software to model airflow within the canopy to investigate the effect of the canopy elements on airflow. This model will further be applied in modelling particle flow within and deposition on orchard canopies as well as to predict drift. This helps in the design, calibration and operation of orchard sprayers for efficient application of pesticides.

\section{MATERIALS AND METHODS}

Two steps were followed to tackle the present work: (1) 3D architectural modelling of orchard canopy, and (2) linking the canopy with CFD software package to simulate airflow.

\section{D Architectural Modelling of Orchard Canopy}

The configurations of the individual elements of plants in a genus follow relatively simple rules that enable the mathematical description of the 'genetic construction plan' of a plant (Muhar, 2001). As presented by Melese Endalew (2006) hereby in this section, the architecture of an orchard tree was modelled using a combined discrete-continuous growth model. ODEs were used to describe time and temperature dependence of the continuous growth functions and discrete events were introduced to indicate when certain threshold of a growth inhibiting hormone $(\mathrm{GIH})$ was reached. The basic unit of the model is an object representing an internode whose life cycle starts as a bud and grows to its final length if the level of GIH is below critical value for the bud to start growing. The growing bud produces GIH which is exported to the lower internodes. At the end of its growth it generates a new active apical bud and a number of (dormant) side buds. The cycle starts over again for each of the newly generated apical and growing buds. Growth of an individual internode is modelled using a sigmoid growth curve given by:

$$
\frac{\mathrm{dL}}{\mathrm{dt}}=\mathrm{k}_{\mathrm{g}}\left(1-\frac{\mathrm{L}}{\mathrm{L}_{\max }}\right)
$$

with $\mathrm{L}[\mathrm{mm}]$ the length of the internode at a given time $t, L_{\max }[\mathrm{mm}]$ the length of a fully grown internode and $\mathrm{k}_{\mathrm{g}}$ [mm/day] the growth rate which is made dependent of temperature. Yearly temperature is simulated assuming a sinusoidal pattern.

This model assumes an endogenous growth control mechanism; production of GIH by the apex that is transported down through the lower internodes inhibiting side buds from growing if the level is greater than a certain threshold value. For a growing bud the GIH turnover is related to the difference between a constant GIH production rate $\left(\mathrm{k}_{\mathrm{p}}\right)$ and part of it exported down to the next lower internode at a rate of $\mathrm{k}_{\mathrm{GIH}} \cdot \mathrm{GIH}$ resulting in:

$$
\frac{\mathrm{dGIH}}{\mathrm{dt}}=\mathrm{k}_{\mathrm{p}}-\mathrm{k}_{\mathrm{GIH}} \cdot \mathrm{GIH}
$$

$\mathrm{k}_{\mathrm{p}}$ ceases to exist by the time the growing bud has reached $\mathrm{L}_{\max }$. GIH turnover in any of the lower internodes is governed by the balance between import from the upper internode(s) and/or growing side bud(s) and export to the lower internode resulting in: 


$$
\frac{\mathrm{dGIH}}{\mathrm{dt}}=\sum_{\mathrm{i}=0}^{\mathrm{n}} \mathrm{k}_{\mathrm{GIH}_{(\mathrm{i})}} \cdot \mathrm{GIH}_{\mathrm{suc}_{(\mathrm{i})}}-\mathrm{k}_{\mathrm{GIH}} \cdot \mathrm{GIH}_{\mathrm{pre}}
$$

where $\mathrm{i}$ is the upper internode under consideration, $\mathrm{n}$ number of upper internodes, $\mathrm{k}_{\mathrm{GIH}}$ $\left[\mathrm{s}^{-1}\right] \mathrm{GIH}$ transport rate constant, and $\mathrm{GIH}_{\text {suc }}$ and $\mathrm{GIH}_{\text {pre }}$ are respectively GIH levels coming from the upper (successor) internodes and leaving to the lower (predecessor) internode with reference to the internode under consideration. The hormone balance is affected by exogenous mechanisms; seasonal temperature changes, affecting $\mathrm{k}_{\mathrm{p}}$, and pruning activities, inducing a new burst of growth after pruning when the hormone levels are locally low again. All side buds remain dormant waiting for their time to come (if ever) to become active and start growing depending on the GIH threshold level. By the use of random factors in the angle under which each internode develops, the final length of an internode, the critical levels of GIH for each bud to induce growth etc., the shape of the final tree can be manipulated. The exact 3D starting and end positions of the internodes are calculated using straightforward trigonometric relationships using the branching angle and the final length of the internode.

Some of the input parameters used in this modelling include, $\mathrm{k}_{\mathrm{g}}$, growth time in years, maximum and minimum stem radius within the growth time, internode length, growth activation energy, $\mathrm{k}_{\mathrm{GIH}}$, critical $\mathrm{GIH}$ level for growth initiation, number of side buds per node, average growth angle of a new internode and others. This model first generates a 3D outline of the tree using internode centrelines and then the solid tree is formed by building conical bodies around these lines. At this stage, random and sensible values were given for the parameters to acquire the desired shape of the tree. In the future, these parameters will be defined based on experimental data and field measurements. The internodes of a branch, represented by conical bodies are defined by their length, bottom and top radius. The radii vary depending on the age of the branch. The $3 \mathrm{D}$ architectural model was implemented using Prosim (Prosim bv, Zoetermeer, NL). Prosim is a software environment for combined discrete-continuous modelling and simulation.

\section{Turbulence Modelling of Airflow within the Canopies}

1. Domain and Boundary Settings. A representative fluid domain with $16.5 \mathrm{~m}$ length, 2 $\mathrm{m}$ width and $8 \mathrm{~m}$ height was used (Fig. 2). Two different trees of about $3 \mathrm{~m}$ height were placed in the domain on the bottom ground (no-slip wall boundary) $0.75 \mathrm{~m}$ and $2.25 \mathrm{~m}$ from the inlet. The two sides of the domain that extend $1 \mathrm{~m}$ from the central xy plane were taken as symmetry planes. Based on the assumption that the log law holds all the way down to the top of the obstacles (Ayotte et al., 1999), initially a logarithmic velocity profile was imposed at the inlet (Fig. 2), which was the simplest and most consistent assumption we could make about conditions on the soil surface below the canopy. Constant gradients were assumed for turbulence kinetic energy $\mathrm{k}$ and its eddy dissipation rate $\varepsilon$ was a function of height $\mathrm{y}$ and the friction velocity $\mathrm{u}_{*}$ within the atmospheric boundary layer (ABL). The outlet and the top of the domain were set pressure and Cartesian velocity outlet boundaries respectively.

2. Meshing and Solution Procedure. Unstructured control volume mesh was used to discretise the computational domain. For meshing, a maximum edge length of $1 \mathrm{~m}$ was defined for the free space with a minimum size of $1 \mathrm{~mm}$ on the surface of the smallest size branches which was reduced to the maximum edge length in 8 layers. This mesh consisted of 3272488 tetrahedral elements uniformly distributed over the domain with a total number of 607385 nodes. The solution converged to a normalized RMS residual of less than $10^{-7}$ for all equations after 500 iterations. Total CPU time of calculation was about 42 hours on an Intel Pentium IV, 3GHz Win2002 workstation with $1 \mathrm{~GB}$ of RAM computer. Cyclic simulation (preceding simulation results used as inputs for proceeding one until the change in the two consecutive simulations is insignificant) was made to model airflow through trees planted in an infinitely long row to represent flow in an orchard field assuming that the field is symmetric along the rows. 
3. Governing Equations. The k- $\varepsilon$ turbulence model is the most popular and standard for computational software packages in engineering and is used in several spray models (e.g. Brown and Sidhamed, 2001; Da Silva et al., 2006). However, its application to model turbulence flow in plant canopies has not given comparable attention; which may be due to the fact that parameterization of the additional source (and/or sink) terms in closure models remains uncertain (Sanz, 2003). In this work, since real 3D canopy structures were introduced into the simulation domain, $\mathrm{k}-\varepsilon$ turbulence model was used without source or sink terms. This might overcome the uncertainty encountered with the parameterization of the sink and source terms. The CFD code used for this modelling was CFX 5.7.1 (ANSYS, Inc., Canonsburg, Pennsylvania, USA). The Reynolds-averaged Navier-Stokes (RANS) equations in their conserved form and $\mathrm{k}-\varepsilon$ turbulence model were used to simulate airflow.

\section{RESULTS AND DISCUSSION}

The tree architectural modelling results are shown in Fig. 1; (a) shows the outline of the canopy as generated in Prosim using centrelines of the tree internodes, (b) the outline converted into solid tree by creating conical bodies around the lines with the internodes as separate parts in the tree, (c) the separate internodes were connected and the geometry was maintained to give one single solid tree. This approach is able to generate more complex tree architectures (Fig. 1d) but such complex tree geometries are difficult to handle in CFD.

Results of normalized average vertical profiles of longitudinal velocity $U$ within the canopies are shown in Fig. 3. U was normalized with the friction velocity $\mathrm{u}_{*}$ for the cyclic simulation and a uniform velocity inlet $U_{\text {in }}$ for the wind tunnel validation. Domain height $\mathrm{Y}$ was normalized with the height of the tree $\mathrm{h}$ for both cases. Fig. 3a shows the velocity profiles captured in the cyclic simulation and it was compared with and is similar to previous works (Gross, 1987; Albertson et al., 2001; Cionco and Ellefsen, 1998). As most researchers in the area agree (e.g. Gross, 1987; Georgiadis et al., 1996) the vertical velocity profiles exhibit three regions (Fig. 3a). The first region is the region above the ground to the height of the tree. This region is known as the canopy flow region as the flow is highly influenced by the presence of the trees and that is why the velocity in this region is too low. The next region is above the height of the trees to twice the height. In this region the flow tends to catch the logarithmic profile and it is called the transition flow region. The third region is the region above twice the height of the trees where the flow is not affected by the trees anymore and the profile becomes back to logarithmic. All the profiles in Fig. 3a are similar and show the three regions confirming that they are qualitatively the same. Some of the quantitative deviations might come from the differences in the shape and density of the trees used in each of the methods. A wind tunnel validation experiment was carried out in Applied Mechanics and Energy Conversion Section, Department of Mechanical Engineering, Catholic University of Leuven using two artificial trees. The artificial trees were converted into their virtual form using 'measure and represent' method and they were put in a simulation domain with the same boundary conditions as in the wind tunnel (uniform velocity inlet and pressure opening outlet with the rest of the boundaries as no slip wall) to model airflow. The vertical velocity profile of simulated Vs measured behind the wake of the trees as shown in Fig. 3b revealed good quantitative agreement with a correlation of $94.9 \%$.

Previously, with the lumped approaches, it was impractical to account explicitly for the spatial variability imposed on the airflow by the complexity of the within-canopy air space. However in reality and as the recent work of Melese Endalew et al. (2006) showed, the interaction of the wind field within the canopy elements produces large amounts of fine-scale velocity reduction in the wakes of canopy elements. It was hardly possible to show these fine-scale reductions using the previous methods but by introducing a real canopy architecture modelled with this method into ANSYS-CFX, it was possible to show the fine scale reductions as shown by velocity contour plot (Fig. 4). All simulations showed an overall reduction of wind speed inside the trees, an accelerated 
flow generally around the trees and specifically around the branches and a wake region in the lee.

\section{CONCLUSIONS}

Although there are several 3D plant architectural modelling algorisms like the Lsystem (Prusinkiewicz and Lindenmayer, 1996) non of them were intended to be linked with CFD to simulate transport properties of air and particles through the canopies. In this work, we have been successful in modelling the architecture of orchard canopies that were compatible to be linked with CFD software package, ANSYS-5.7 to simulate airflow within the canopies. The 3D plant canopy architecture satisfactorily represented the main features of the plant such as branching, branching pattern and branch distribution at the canopy level. The 3D representation of the canopies made it possible to investigate the detailed effects of the canopy elements on turbulent airflow. The results obtained in this approach agreed qualitatively with previous works and quantitatively with wind tunnel validation result. Therefore, the approach may potentially address some of the problems attributed to porous media approaches used to model air and particle flow within orchard canopies and to predict drift. However, leaves were not included in the tree model for two reasons. The first was to minimize computational complexity, especially considering the problem leaf orientation can bring during meshing the domain. Excluding the leaves may also help to approach the 'worst case scenario' in drift prediction. The second was due to lack of knowledge on how leaf growth and orientation (leaf midrib curvature and azimuth) depend on physiological processes and environmental conditions. It will be important to consider leaves in future work to take into account their effects to the atmospheric turbulence, provided that the computational capability advances to handle such complexities.

\section{ACKNOWLEDGEMENT}

The Flemish and Belgium governments are gratefully acknowledged for financial support (S-6209 and IWT-040708-05/591). Pieter Verboven is postdoctoral researcher of the Fund for Scientific Research - Flanders (FWO-Vlaanderen).

\section{Literature Cited}

Albertson, J.D., Katul, G.G. and Wiberg, P. 2001. Relative importance of local and regional controls on coupled water, carbon, and energy fluxes. Advances in Water Resources 24:1103-1118.

Ayotte, K.W., Finnigan, J.J. and Raupach, M.R. 1999. A second-order closure for neutrally stratified vegetative canopy flows. Boundary-Layer Meteorol. 90:189-216.

Birch, C.J., Andrieu, B., Fournier, C., Vos, J. and Room, P. 2003. Modelling kinetics of plant canopy architecture - concepts and applications. European J. Agron. 19:519-533.

Brown, R.B. and Sidahmed, M.M. 2001. Simulation of spray dispersal and deposition from a forestry airblast sprayer - part II: droplet trajectory model. Trans. ASAE 44(1):11-17.

Cionco, R.M. and Ellefsen, R. 1998. High resolution urban morphology data for urban wind flow modelling. Atmospheric Environ. 32:7-17.

Costes, E., Sinoquet, H., Godine, C. and Kelner, J.J. 1999. 3D digitizing based on tree topology: application to study variability of apple quality within the canopy. Acta Hort. 499:271-280.

Da Silva, A., Sinfort, C., Tinet, C., Pierrat, D. and Huberson, S. 2006. A Lagrangian model for spray behaviour within vine canopies. J. Aerosol Sci. 37:658-674.

De Visser, P.H.B., Marcelis, L.F.M., Van der Heijden, G.W.A.M., Vos, J., Struik, P.C. and Evers, J.B. 2002. 3D modelling of plants: a review, report of the virtual plant network. Plant Research International B.V. Wageningen, Netherlands, Report 52. p.44.

Georgiadis, T., Dalpane, E., Rossi, F. and Nerozzi, F. 1996. Orchard-atmosphere physical exchanges: modelling the canopy aerodynamics. Acta Hort. 416:177-182.

Godin, C. 2000. Representing and encoding plant architecture: A review. Ann. Forest. Sci. 
$57: 413-438$.

Gross, G. 1987. A numerical study of the air flow within and around a single tree. Boundary-Layer Meteorol. 40:311-327.

Melese Endalew, A., Hertog, M., Verboven, P., Baetens, K., Delele, M.A., Ramon, H. and Nicolaï, B.M. 2006. Modelling airflow through 3D canopy structure of orchards. International advances in pesticide application 2006. Aspects Appl. Biol. 77:465-472.

Muhar, A. 2001. Three-dimensional modelling and visualisation of vegetation for landscape simulation. Landscape and Urban Planning 54:5-17.

Prusinkiewicz, P. 1998. Modeling of spatial structure and development of plants: a review. Scientia Hort. 74:113-149.

Prusinkiewicz, P. and Lindenmayer, A. 1996. The algorithmic beauty of plants. SpringerVerlag, New York. p.240.

Sanz, C. 2003. A note on k- $\varepsilon$ modelling of vegetation canopy air-flows. Boundary-Layer Meteorol. 108:191-197.

Sievänena, R., Nikinmaa, E., Nygren, P., Ozier-Lafontaine, H., Perttunen, J. and Hakula, H. 2000. Components of functional-structural tree models. Ann. Forest. Sci. 57:399412.

Sinoquet, H., Rivet, P. and Godine, C. 1997. Assessment of the three-dimensional architecture of walnut trees using digitizing. Silva Fennica 31(3):265-273.

Thom, A.S. 1971. Momentum absorption by vegetation. Quarterly J. Royal Meteorol. Soc. 97:414-428.

Xu, Z.G., Walklate, P.J. and McLeod, A.R. 1997. Numerical study of a full-size free-air fumigation system. Agri. Forest Meteorol. 85:159-170.

\section{Figures}

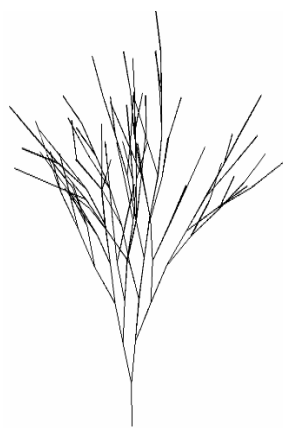

(a)

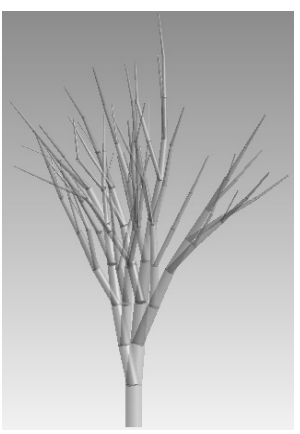

(b)

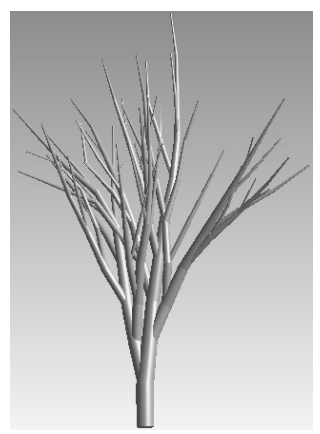

(c)

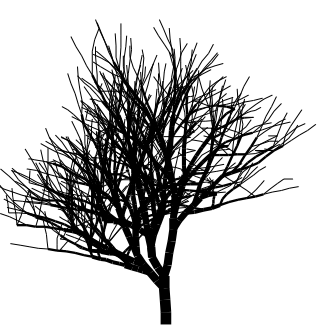

(d)

Fig. 1. 3D canopy architectural modelling results; (a) tree outline as obtained with Prosim, (b) solid tree with internodes as separate geometries, (c) the internodes merged to make one single tree and (d) a more complex tree. 


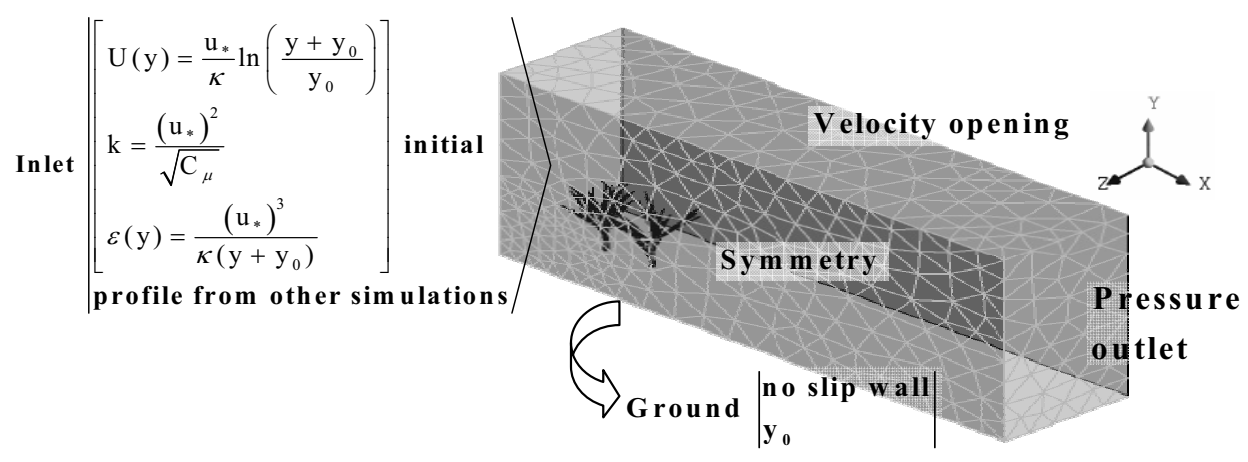

Fig. 2. Boundary settings and unstructured mesh on the surface of the fluid domain.

(a)

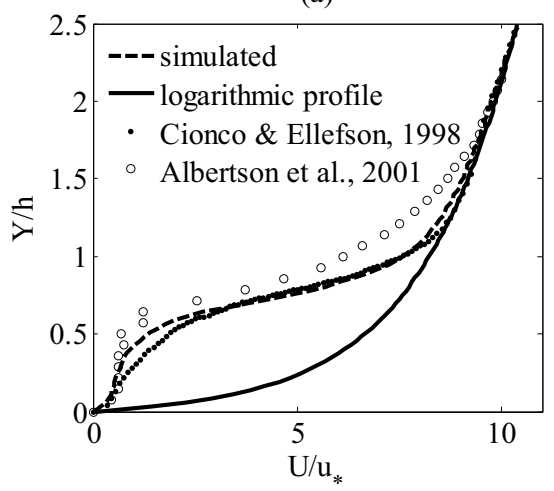

(b)

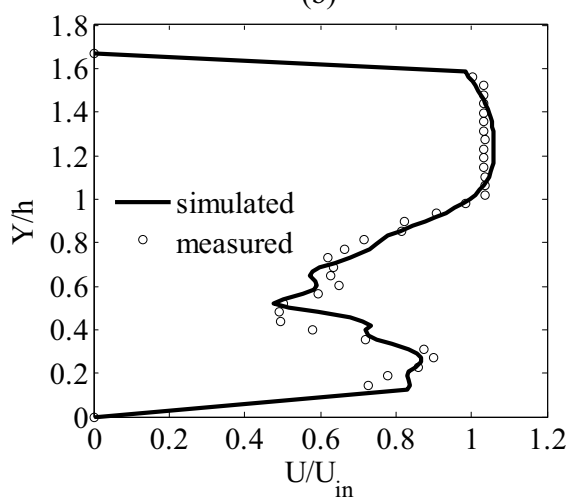

Fig. 3. Normalized vertical profiles of the longitudinal velocity within the canopies: (a) wind tunnel validation experiment compared with simulation and (b) cyclic simulation compared with previous works.

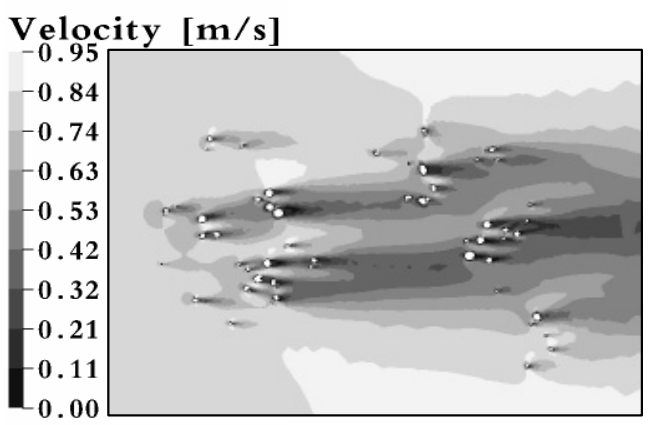

Fig. 4. Velocity contour on a horizontal plane at $1.75 \mathrm{~m}$ height. 
